\title{
Thermal, viscoelastic, and mechanical properties of DCPD-containing polymers
}

\author{
Marta Worzakowska
}

$3^{\text {rd }}$ Joint Czech-Hungarian-Polish-Slovak Thermoanalytical Conference Special Chapter

(c) The Author(s) 2011. This article is published with open access at Springerlink.com

\begin{abstract}
The thermal, viscoelastic, and mechanical properties of cured dicyclopentadiene (DCPD)-containing polymers prepared from novel DCPD-modified unsaturated epoxypolyesters and styrene were evaluated. This was accomplished using thermogravimetric analysis, differential scanning calorimetry, dynamic mechanical analysis, threepoint bending test, and Brinell's hardness. The thermal, viscoelastic, and mechanical properties of DCPD-containing polymers were strongly dependent on chemical structure. The cross-linking density $\left(v_{\mathrm{e}}\right)$ of obtained networks increased with increasing content of carbon-carbon double bonds in the poly(ester) structure. In addition, the introduction of DCPD rings into the poly(ester) structure increased the rigidity of the molecular backbone. It resulted in obtaining polymers which showed great improvement in mechanical properties including remarkably higher storage modulus $\left(E_{20}^{\prime}{ }^{\circ} \mathrm{C}\right)$, flexural modulus at bending $\left(E_{\bmod }\right)$, hardness, lower extension at maximum force $\left(\varepsilon-F_{\max }\right)$, as well as higher thermal stability. These good properties make these materials highly promising as potential candidates for structural applications.
\end{abstract}

Keywords Dicyclopentadiene - Polyesters ·

Thermal properties · Mechanical properties

\section{Introduction}

The properties of cured polymers largely depend on the nature of chemical structure of the starting resins. The

M. Worzakowska ( $\square)$

Department of Polymer Chemistry, Faculty of Chemistry,

Maria Curie-Sklodowska University, Gliniana 33 Str.,

20-614 Lublin, Poland

e-mail: marta.worzakowska@ poczta.umcs.lublin.pl incorporation of various types of moieties into the polymer backbone allows tailoring and preparation of various resins for different applications. The introduction of cycloaliphatic groups into polymer structure often improves the thermal stability, mechanical performance, transparency, and glass transition temperature. The flexibility of cured products obtained from these materials was greater than that of similar products prepared from aliphatic-based polymers [1-4]. One of the compounds, which can be widely applied as a comonomer for polymer synthesis is dicyclopentadiene (DCPD) due to their availability, low cost, and reactivity [5-8]. DCPD is the Diels-Alder reaction dimmer of cyclopentadiene, mainly obtained as a by-product in the olefin cracking process [8]. The difference in reactivity between the norbornene and cyclopentene double bonds in DCPD allows preparing their derivatives (ester monomers or secondary alcohols) which can be successfully used as an intermediate for various syntheses, e.g., production of less expensive polyesters which are characterized by reduced shrinkage during cure $[8,9]$. In addition, DCPD and their based products can be easily oxidized with the use of peracids forming their epoxy derivatives [10]. The resulting epoxides display high reactivity attributed to the high ring strain present in the epoxycyclopentenyl ring system. The cured epoxy DCPDbased resins possess excellent rigidity, mechanical strength, high thermal stability, electrical properties after curing, high dimensional stability, and high resistant to UV light. They are attractive materials for wide industrial applications. They are used as paints, coatings, matrices, packing materials, outdoor applications, etc. [11-14].

In this article, the establishment of the thermal, viscoelastic, and mechanical properties of cured DCPD-containing polymers prepared from novel DCPD-modified unsaturated epoxypolyesters and styrene (ST) is described. This was accomplished using thermogravimetric analysis 
(TG), differential scanning calorimetry (DSC), dynamic mechanical analysis (DMA), three-point bending test, and Brinell's hardness. The influence of poly(ester) structure on cross-linking density $\left(v_{\mathrm{e}}\right), \operatorname{tg} \delta_{\max }, \operatorname{tg} \delta_{\max }$ height, storage modulus $\left(E_{20{ }^{\circ} \mathrm{C}}^{\prime}\right)$, hardness, flexural modulus at bending $\left(E_{\text {mod }}\right)$, deflection at maximum force $\left(\varepsilon-F_{\max }\right)$, as well as the thermal stability of obtained polymer networks was evaluated.

\section{Experimental}

\section{Materials}

Dicyclopentadiene, maleic acid (MA), 1,4-cyclohexanodimetanol (CHDM), cyclohex-4-ene-1,2-dicarboxylic anhydride (THPA), maleic anhydride (BM), and $40 \%$ peracetic acid were obtained from Merck-Schuchardt, Germany. Benzoyl peroxide (BPO) was supplied by Fluka, Switzerland. ST, hydroquinone, and xylene were delivered by POCh, Poland. Butylstannoic acid (catalyst) was from Alkema Inc., USA. All reagents were used as received.

Synthesis of DCPD-modified unsaturated epoxypolyesters

DCPD (1.07 mol), MA (1 mol), and hydroquinone $(0.035$ mass $\%)$ were placed into a $500-\mathrm{mL}$, three-necked, round-bottomed flask equipped with a mechanical stirrer, a thermometer, and a condenser. The mixture was stirred vigorously at $135{ }^{\circ} \mathrm{C}$ for $2.5 \mathrm{~h}$. In this stage, the acidic ester of DCPD was formed. After being allowed to cool, the ester was treated with THPA $(0.5 \mathrm{~mol})$, catalyst $(0.01 \mathrm{mass} \%)$, suitable amounts of BM, and CHDM, as shown in Table 1. The resulting mixture was heated at $150{ }^{\circ} \mathrm{C}$ for $1 \mathrm{~h}$ and then at $180{ }^{\circ} \mathrm{C}$ until the acid value dropped below $3 \mathrm{mg}$ $\mathrm{KOH} \mathrm{g}^{-1}$. Acid value defined as a number of $\mathrm{mg} \mathrm{KOH}$ required for titration of $1 \mathrm{~g}$ of a sample was determined by titration method. During the reaction, water was removed continuously from the system by azotropic distillation (xylene). After that, xylene was distilled under reduced pressure and the obtained product was chemically modified in order to prepare novel DCPD-modified unsaturated epoxypolyesters according to a previously reported procedure [15-17]. The theoretical structure of novel DCPDmodified unsaturated epoxypolyesters is presented in Scheme 1. Their chemical structure was confirmed based on ${ }^{1} \mathrm{H}$ NMR and FTIR analyses, as shown in Figs. 1 and 2.

Characterization of novel DCPD-modified unsaturated epoxypolyesters

Fourier transform infrared (FTIR) spectra were obtained by using a Perkin-Elmer 1725 X FTIR spectrophotometer in the $400-4,000 \mathrm{~cm}^{-1}$ wavenumber range using $\mathrm{KBr}$ pellets.

Proton nuclear magnetic resonance ( ${ }^{1} \mathrm{H}$ NMR) spectra were recorded on an NMR Brucker-Avance 300 MSL (Germany) spectrometer at $300 \mathrm{MHz}$ with deuterated chloroform $\left(\mathrm{CDCl}_{3}\right)$ as the solvent. ${ }^{1} \mathrm{H}$ NMR chemical shifts in parts per million (ppm) were reported downfield from $0.00 \mathrm{ppm}$ using tetramethylsilane as an internal reference.

\section{Characterization of DCPD-containing polymers}

The calorimetric measurements were carried out in the Netzsch DSC 204 calorimeter (Germany) operating in a dynamic mode. The dynamic scans were performed at a heating rate of $10{ }^{\circ} \mathrm{C} \mathrm{min}-1$ from room temperature to a maximum of $500{ }^{\circ} \mathrm{C}$ under nitrogen atmosphere $\left(30 \mathrm{~mL} \mathrm{~min}^{-1}\right)$. As a reference an empty aluminum crucible was used. The characteristic temperatures during

Table 1 Composition of the reaction mixture

\begin{tabular}{llllll}
\hline $\begin{array}{l}\text { Poly(ester) } \\
\text { no. }\end{array}$ & $\begin{array}{l}\text { DCPD/ } \\
\text { mol }\end{array}$ & $\begin{array}{l}\text { MA/ } \\
\text { mol }\end{array}$ & $\begin{array}{l}\text { THPA/ } \\
\text { mol }\end{array}$ & $\begin{array}{l}\text { BM/ } \\
\text { mol }\end{array}$ & $\begin{array}{l}\text { CHDM/ } \\
\text { mol }\end{array}$ \\
\hline 1 & 1.07 & 1 & 0.5 & 0 & 1.07 \\
2 & 1.07 & 1 & 0.5 & 0.5 & 1.61 \\
3 & 1.07 & 1 & 0.5 & 1 & 2.15 \\
\hline
\end{tabular}

Scheme 1 The theoretical structure of novel DCPDmodified unsaturated epoxypolyesters

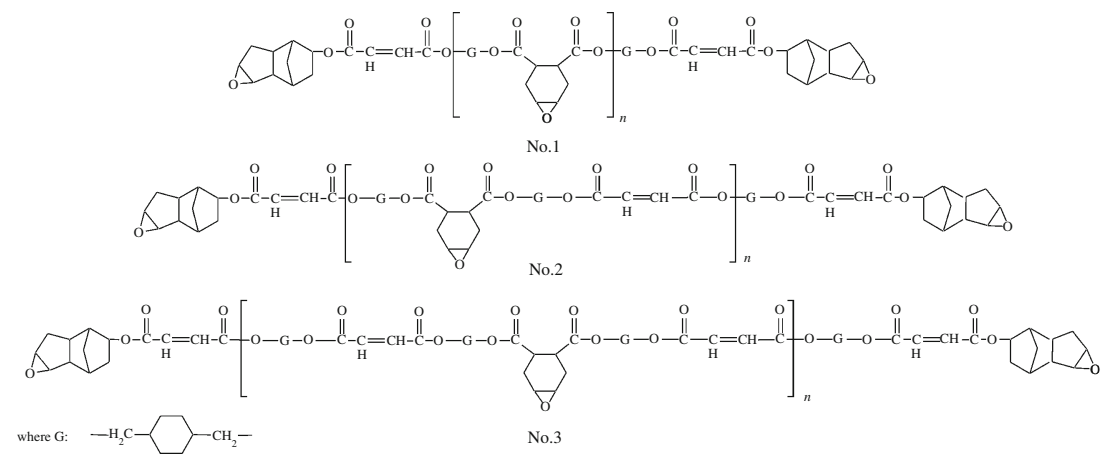


Fig. $1{ }^{1} \mathrm{H}$ NMR spectra of novel DCPD-modified unsaturated epoxypolyesters

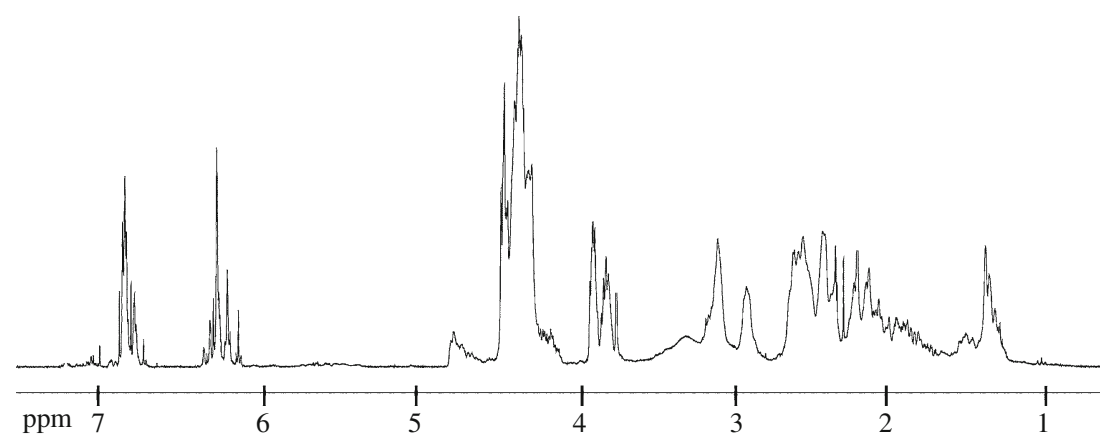

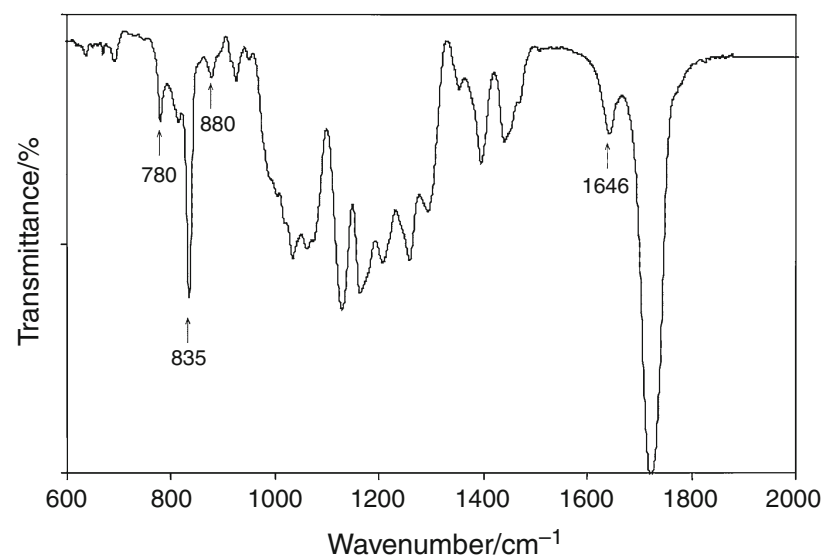

Fig. 2 FTIR spectra of novel DCPD-modified unsaturated epoxypolyesters

degradation $(T)$ and $\Delta H$ values obtained by the integration of the degradation peaks were evaluated.

Thermogravimetric experiments were carried out on a STA 449 Jupiter F1, Netzsch (Germany) under the following operational conditions: heating rate of $10{ }^{\circ} \mathrm{C} \mathrm{min}{ }^{-1}$, a helium atmosphere $\left(30 \mathrm{~mL} \mathrm{~min}^{-1}\right)$, the temperature range of $30-800{ }^{\circ} \mathrm{C}$, the sample mass of $\sim 10 \mathrm{mg}$. As a reference empty $\mathrm{Al}_{2} \mathrm{O}_{3}$ crucible was used. The temperature of 10,20 , and $50 \%$ of mass loss $\left(T_{10 \%}, T_{20 \%}, T_{50 \%}\right)$, the temperature of the maximum rate of mass loss $\left(T_{\max }\right)$ and the residual mass at $800{ }^{\circ} \mathrm{C}\left(\mathrm{rm}_{800}{ }^{\circ} \mathrm{C}\right)$ were determined.

Dynamic mechanical analysis measurements were performed using Dynamic Mechanical Analyzer (DMA) Q 800 TA Instruments (USA). Tests were conducted with a double Cantilever device with a support span of $35 \mathrm{~mm}$, calibrated according to the producer's recommendation. The temperature scanning from room temperature until the sample become too soft to be tested with a constant heating rate of $4{ }^{\circ} \mathrm{C} \mathrm{min}{ }^{-1}$ at an oscillation frequency of $10 \mathrm{~Hz}$ was performed. The rectangular profile of the samples $10 \mathrm{~mm}$ wide and $4 \mathrm{~mm}$ thick was used. The storage modulus $\left(E_{20{ }^{\circ} \mathrm{C}}^{\prime}\right)$, glass transition temperature ( $\alpha$-relaxation) identified as a maximum of the tangent delta $\left(\operatorname{tg} \delta_{\max }\right), \operatorname{tg} \delta_{\max }$ height, crosslinking density $\left(v_{\mathrm{e}}\right)$, and FWHM values were qualified [18].
Hardness according to Brinell was determined by means of a hardness tester HPK and calculated based on the following equation: Hardness/MPa $=F_{1} \times 0.098066$, where $F_{1}$ is the strength of a pressure under definite load.

Flexural properties were measured in a tree-point bending test using a Zwick/Roell Strength Machine (model Z010, Germany). The specimen dimensions were $10 \mathrm{~mm}$ wide and $4 \mathrm{~mm}$ thick. The measurements were carried out at room temperature with a crosshead speed of $2 \mathrm{~mm} \mathrm{~min}^{-1}$ using a support span of $64 \mathrm{~mm}$. At least five specimens were tested for each sample to evaluate the flexural properties. The flexural modulus at bending $\left(E_{\bmod }\right)$ and the deflection at maximum force $\left(\varepsilon-F_{\max }\right)$ were qualified.

Curing procedure

The novel DCPD-modified unsaturated epoxypolyesters were diluted in ST making solutions of the concentration: 30 mass \% of monovinyl monomer. The mixture of stoichiometric amount of hexahydrophthalic anhydride (hardener of epoxy groups) and 1.0 mass $\%$ of BPO (thermal initiator) was used as a curing system. The obtained ST compositions after degassing were placed in a glass mold, conditioned in the temperature range of $60-120^{\circ} \mathrm{C}$ and then post-cured at $160-180{ }^{\circ} \mathrm{C}$, until no further exothermic peak maxima connected with additional effect of postcuring was seen from DSC curves.

\section{Results and discussion}

Characterization of novel DCPD-modified unsaturated epoxypolyesters

The novel DCPD-modified unsaturated epoxypolyesters were prepared during three stage process. First, the acidic ester derived from DCPD and MA was formed. Then, the polycondensation process of the acidic ester of DCPD, THPA, different amounts of BM and CHDM was performed. Finally, the obtained DCPD-modified unsaturated polyesters were chemically modified in order to prepare novel DCPD- 
modified unsaturated epoxypolyesters. Their structure was confirmed based on ${ }^{1} \mathrm{H}$ NMR and FTIR analyses. ${ }^{1} \mathrm{H}$ NMR spectrum of DCPD-modified unsaturated epoxypolyester is shown in Fig. 1. The resonance signals at $2.8-3.2 \mathrm{ppm}$ connected with the protons in surroundings of epoxy groups are visible. Also, the resonance signals attributed to cis-trans units of BM at 6.20-6.40 ppm (cis unit) and 6.80-6.95 ppm (trans unit) are clearly observed. Figure 2 presents the example FTIR spectra of DCPD-modified unsaturated epoxypolyester. The strong absorption bands at $780-880 \mathrm{~cm}^{-1}$ corresponding to oxirane ring vibration groups and $\mathrm{C}=\mathrm{C}$ stretching vibration for carbon-carbon double bonds of $\mathrm{BM}$ at $1,646 \mathrm{~cm}^{-1}$ are indicated. The analyses unambiguously confirmed the formation of desirable product.

Thermal, viscoelastic, and mechanical properties of DCPD-containing polymers

The DMA results and the results obtained based on mechanical studies of cured DCPD-containing polymers are depicted in Table 2. The results show that the $\operatorname{tg} \delta_{\max }$, transition breadth and the temperature of the $\operatorname{tg} \delta_{\max }$ of each polymer is determined by the cross-linking density, as is the rubbery modulus [19-21]. An increase in the cross-linking density $\left(v_{\mathrm{e}}\right)$ is connected with the decrease in molecular mobility of the polymer chains and thus with the increase of the glass transition temperature (described as maximum of the temperature of $\left.\operatorname{tg} \delta_{\max }\right)$, the storage modulus $\left(E_{20{ }^{\circ} \mathrm{C}}^{\prime}\right)$, flexural modulus at bending $\left(E_{\mathrm{mod}}\right)$, hardness and decrease of deflection at maximum force $\left(\varepsilon-F_{\max }\right)$. In addition, DCPD-containing polymers prepared in the presence of poly(ester) No. 3 are characterized by better viscoelastic and mechanical properties comparing to DCPD-containing polymers prepared in the presence of poly(esters) Nos. 1 and 2. The higher values of $E_{20}^{\prime}{ }^{\circ}, v_{\mathrm{e}}, \operatorname{tg} \delta_{\text {max }}, E_{\text {mod }}$, hardness and lower values of $t g \delta_{\max }$ height, FWHM and $\varepsilon-F_{\max }$ of cured DCPD-containing polymers based on poly(ester)
No. 3 are observed. It is probably connected with the higher concentration of the double bonds in the structure of poly(ester) No. 3. The higher content of the unsaturated bonds in the poly(ester) structure causes obtaining more densely cross-linked polymer networks with consequently larger rigidity and more mechanical stability.

The thermal properties of DCPD-containing polymers are shown in Table 3. TG analysis of obtained DCPDcontaining polymers shows the differences in their thermal behavior. The higher values of the temperature of 10, 20, and $50 \%$ of mass loss $\left(T_{10 \%}, T_{20 \%}, T_{50 \%}\right)$ and higher percent of the residual mass at $800{ }^{\circ} \mathrm{C}\left(\mathrm{rm}_{800}{ }^{\circ} \mathrm{C}\right)$ for DCPDcontaining polymers prepared based on poly(ester) No. 3 are observed. It confirms the formation of more thermally stable polymer networks due to their higher cross-linking density which is connected with higher content of the unsaturated units in the poly(ester) structure. In addition two maximum rate peaks $\left(T_{\max }\right)$ appear in DTG curves, as shown in Fig. 3. It suggests that the degradation of all cured DCPD-containing polymers runs through at least two degradation steps. The first, large decomposition peak $\left(T_{\max 1}\right)$ is observed in the temperature range of $290-390{ }^{\circ} \mathrm{C}$ with the maximum mass loss $\left(T_{\max 1}\right)$ at $362-365{ }^{\circ} \mathrm{C}$, the second one at $420-424{ }^{\circ} \mathrm{C}$. These decomposition peaks are directly connected with the degradation of linkages present in the poly(ester) structure and formed during the cure process. Also, based on DSC curves, two endothermal signals are indicated, as shown in Fig. 3. The highest $\Delta H_{1}$ values (obtained by the integration of first degradation peak) are observed for polymer networks prepared in the presence of poly(ester) No. 3. It indicates on the production of more cross-linked networks and confirms earlier observations. Also, the $\Delta H_{2}$ decreases as the content of the unsaturated bonds in the poly(ester) structure increases. Probably, the second, small endothermal peak is due to the degradation of polystyrene formed during the cross-linking process (the degradation of pure polystyrene happened at about $418{ }^{\circ} \mathrm{C}$ in the studied experimental conditions).

Table 2 Viscoelastic and mechanical properties of DCPD-containing polymers

\begin{tabular}{|c|c|c|c|c|c|c|c|c|}
\hline Poly(ester) no. & $E_{20^{\circ} \mathrm{C}}^{\prime} / \mathrm{MPa}$ & $\operatorname{tg} \delta_{\max }$ & $\operatorname{tg} \delta_{\max } /{ }^{\circ} \mathrm{C}$ & $v_{\mathrm{e}} \times 10^{-3} / \mathrm{mol} / \mathrm{cm}^{3}$ & $\mathrm{FWHM} /{ }^{\circ} \mathrm{C}$ & $E_{\text {mod }} / \mathrm{GPa}$ & $\varepsilon-F_{\max } 1 \%$ & Hardness/MPa \\
\hline 1 & 3,150 & 0.453 & 120 & 0.262 & 36 & 3.30 & 2.58 & 142 \\
\hline 2 & 3,300 & 0.325 & 132 & 0.385 & 30 & 3.45 & 1.65 & 165 \\
\hline 3 & 3,540 & 0.216 & 145 & 0.450 & 22 & 3.70 & 0.86 & 178 \\
\hline
\end{tabular}

Table 3 Thermal properties of DCPD-containing polymers

\begin{tabular}{lllllllllll}
\hline Poly(ester) no. & $T_{10 \%} /{ }^{\circ} \mathrm{C}$ & $T_{20 \%} /{ }^{\circ} \mathrm{C}$ & $T_{50 \%} /{ }^{\circ} \mathrm{C}$ & $\mathrm{rm}_{800}{ }^{\circ} \mathrm{C} / \%$ & $T_{\max 1} /{ }^{\circ} \mathrm{C}$ & $T_{\max 2} /{ }^{\circ} \mathrm{C}$ & $T_{1} /{ }^{\circ} \mathrm{C}$ & $T_{2} /{ }^{\circ} \mathrm{C}$ & $\Delta H_{1} / \mathrm{J} \mathrm{g}{ }^{-1}$ & $\Delta H_{2} / \mathrm{J} \mathrm{g}$ \\
\hline 1 & 290 & 320 & 360 & 1.1 & 362 & 420 & 360 & 418 & 396.7 & 33.9 \\
2 & 300 & 335 & 365 & 2.0 & 364 & 424 & 362 & 420 & 465.8 & 18.5 \\
3 & 310 & 330 & 365 & 3.2 & 365 & 423 & 363 & 420 & 545.8 & 5.2 \\
\hline
\end{tabular}



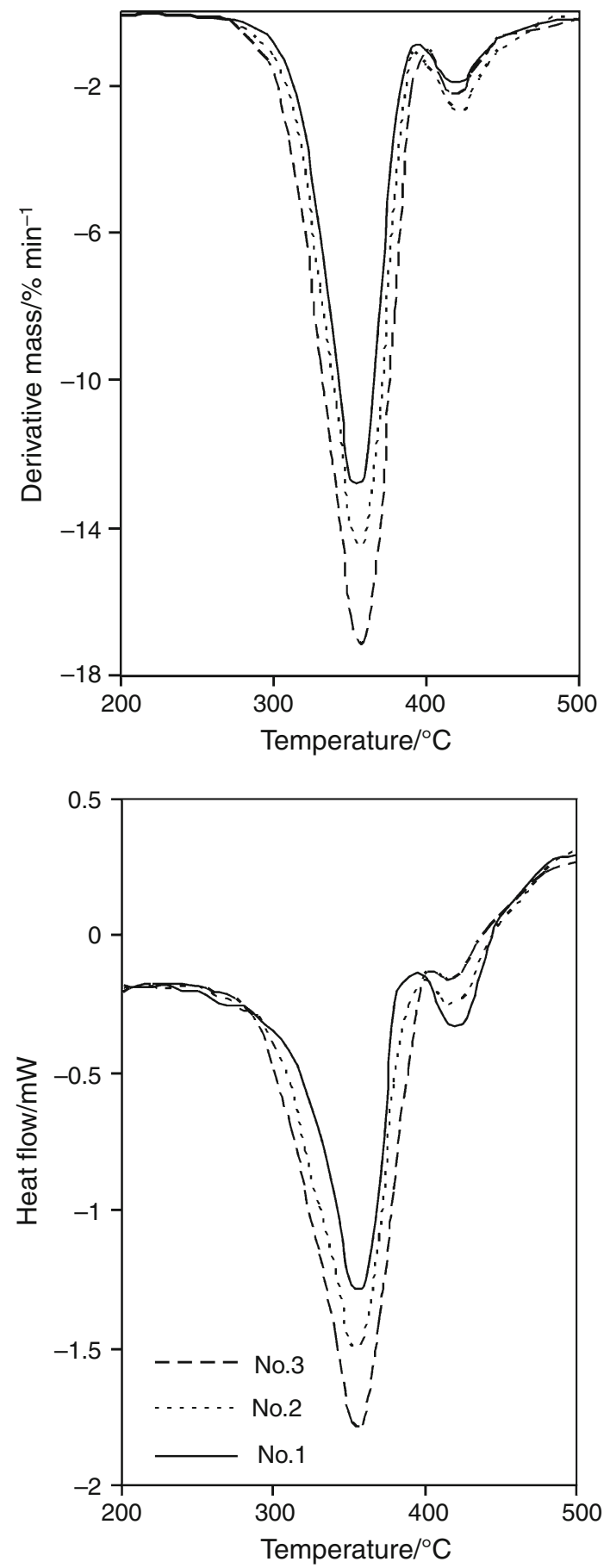

Fig. 3 DTG and DSC curves of DCPD-containing polymers

\section{Conclusions}

The thermal, viscoelastic, and mechanical properties of cured DCPD-containing polymers prepared from novel DCPD-modified unsaturated epoxypolyesters and ST were evaluated. This was accomplished using TG, DSC, DMA, three-point bending test, and Brinell's hardness. The studies confirmed that the DCPD-containing polymers prepared in the presence of novel DCPD-modified unsaturated epoxypolyesters and ST were characterized by improved thermal, viscoelastic, and mechanical properties comparing to those previously studied [15, 16, 22]. Such properties make these materials highly promising as potential candidates for structural applications.

Open Access This article is distributed under the terms of the Creative Commons Attribution Noncommercial License which permits any noncommercial use, distribution, and reproduction in any medium, provided the original author(s) and source are credited.

\section{References}

1. Jung IK, Lee KH, Chin IJ, Yoon JS, Kim MN. Properties of biodegradable copolyesters of succinic acid-1,4-butanediol/succinic acid-1,4-cyclohexanedimethanol. J Appl Polym Sci. 1999;72: 553-8.

2. Ni H, Daum JL, Thiltgen PR, Soucek MD, William J, Simonsick Jr. Cycloaliphatic polyester-based high-solids polyurethane coatings: II. The effect of difunctional acid. Prog Org Coat. 2002; 45:49-58.

3. Johnson LK, Sade WT. New monomers for polyester. Powder coating resins. J Coat Technol. 1993;65:19-26.

4. Sun YM, Wang CS. Preparation and characterization of poly(ethylene-1,4-cyclohexanedimethylene arylate). Eur Polym J. 1999;35:1087-96.

5. Wang T, Wan PY, Yu QP, Yu M. Synthesis and characterization of dicyclopentadiene-cresol epoxy resin. Polym Bull. 2008;59: 787-93.

6. Hsiue GH, Shiao SJ, Wei HF, Kuo WJ, Shai YA. Novel phosphorus-containing dicyclopentadiene-modified phenolic resins for flame retardancy applications. J Appl Polym Sci. 2001;79:342-9.

7. Zhang X, Zhang Z, Xia X, Zhang Z, Xu W, Xiong Y. Synthesis and characterization of a novel cycloaliphatic epoxy resin starting from dicyclopentadiene. Eur Polym J. 2007;43:2149-54.

8. Johnson KG, Yang LS. Preparation, properties and applications of unsaturated polyesters. In: Scheirs J, Long TE, editors. Modern polyesters: chemistry and technology of polyesters and copolyesters. New York: Wiley; 2003. Chap. 21, p. 699-713.

9. Crivello JV, Soyoung S. Synthesis and cationic photopolymerization of novel monomers based on dicyclopentadiene. Chem Mater. 2000;12:3674-80.

10. McGary CW, Patrick CT, Smith PL. Resins from endo-dicyclopentadiene dioxide. J Appl Polym Sci. 1963;7:1-14.

11. Lubin G. Handbook of composites. New York: Van Nostrand Reinhold; 1982.

12. LeMay JD, Kelley FN. Structure and ultimate properties of epoxy resins. Adv Polym Sci. 1985;78:115-48.

13. Yang Thomas CK, Stanley HY, Tsai, Wang SF, Juan CH. Dielectric and thermal studies of inorganic microfillers on polymer microwave substrates-metallocene cyclic olefin copolymers (COC). Compos Sci Technol. 2002;62:655-61.

14. Lee SM. Epoxy resins. New York: Mercel Dekker; 1988. p. 860-5.

15. Worzakowska M. Studies on the cure reaction and thermal properties of NADIC/or PA modified unsaturated (epoxy) polyesters. J Therm Anal Calorim. 2010;99:599-608.

16. Worzakowska M. Succinic/or glutaric anhydride modified unsaturated (epoxy) polyesters. J Therm Anal Calorim. 2010;101:685-93.

17. Worzakowska M. The influence of tertiary aromatic amines on the BPO initiated cure of unsaturated epoxy polyesters with styrene studied by non-isothermal DSC. J Therm Anal Calorim. 2011;105:987-94. 
18. Ward IM. Mechanical properties of solid polymer. London: Wiley; 1971.

19. Shibayama K, Suzuki Y. Effect of crosslinking density on the viscoelastic properties of unsaturated polyesters. J Polym Sci A. 1965;3:2637-51.

20. Nielsen LJ. Cross-linking-effects on physical properties of polymers. Macromol Sci Rev Macromol Chem. 1969;C3(1): 69-103.
21. Dusek K. Network formation in curing of epoxy resins. Adv Polym Sci. 1985;78:1-59.

22. Worzakowska M. Chemical modification of unsaturated polyesters. Influence of polyester's structure on thermal and viscoelstic properties of low styrene content copolymers. J Appl Polym Sci. 2009; 114:720-31. 